\title{
Implicit versus Explicit Knowledge in Dialogical Logic
}

\author{
Manuel Rebuschi \\ L.P.H.S. - Archives H. Poincaré \\ Université de Nancy 2 \\ manuel.rebuschi@univ-nancy2.fr
}

[The final version of this paper is published in: O. Majer et al. (eds.), Games: Unifying Logic, Language, and Philosophy, Dordrecht, Springer, 2009, 229-246.]

\begin{abstract}
A dialogical version of (modal) epistemic logic is outlined, with an intuitionistic variant. Another version of dialogical epistemic logic is then provided by means of the S4 mapping of intuitionistic logic. Both systems cast new light on the relationship between intuitionism, modal logic and dialogical games.
\end{abstract}

\section{Introduction}

Two main approaches to knowledge in logic can be distinguished [⿰]. The first one is an implicit way of encoding knowledge and consists in an epistemic interpretation of usual logic. This is for instance the case of Intutionistic logics, especially of the socalled BHK interpretation of it. There, assertion is assimilated to provability, negation to the provability of contradiction, etc. The second approach is what is known, since Hintikka's seminal work [], as (modal) epistemic logic. In this case, knowledge is explicitely supported by modal operators.

The aim of the paper is to show the specific insight provided by dialogical games on this distinction. In section 1 , I will introduce dialogical versions of classical and intuitionistic PL and a dialogical version of modal epistemic logic. In section 2 and 3, two combinations of implicit and explicit epistemic logic are accounted for: Intuitionistic modal logic, and a modal embedding of intuitionistic logic. In section 4, other issues connected with the implementation of epistemic logic in the dialogical frame are raised and briefly discussed.

\section{Dialogical Epistemic Logic (DEL) in a Nut- shell}

Thanks to a straighforward transposition of Rahman \& Rückert's Dialogical Modal Logic 12], one obtains a Dialogical Epistemic Logic (hereafter DEL). For that purpose, 
several kinds of rules have to be stated: structural and particle rules for propositional logic and for modal epistemic logic. As will be shown, modal logic only requires a simple extension of rules for propositional logic.

\subsection{Propositional Logic}

In a dialogical game, two players argue about a thesis: The proponent $\mathbf{P}$ defends it against the attacks of the opponent $\mathbf{O}$. As usually in game semantics, something interesting appears when the proponent has a winning strategy, i.e. if she can defend the proposition against any attack from the opponent. Here the interesting result is that one is guaranteed that the proposition is logically true or valid - whereas with in GTS for instance, the existence of a winning strategy means that the challenged proposition is true simpliciter.

Particle Rules The meaning of each logical constant is given through a particle rule which determines how to attack and defend a formula whose main connective is the constant in question. The set, PartRules, of particle rules for disjunction, conjunction, subjunction and negation is recapitulated in the following table:

\begin{tabular}{|c|c|c|}
\hline & Attack & Defence \\
\hline$A \vee B$ & $?$ & $\begin{array}{c}A, \text { or } B \\
\text { (The defender chooses) }\end{array}$ \\
\hline$A \wedge B$ & $\begin{array}{c}?_{L}, \text { or } ?_{R} \\
\text { (The attacker chooses) }\end{array}$ & $\begin{array}{c}A, \text { or } B \\
\text { (respectively) }\end{array}$ \\
\hline$A \rightarrow B$ & $A$ & $B$ \\
\hline$\neg A$ & $\bar{A}$ & $\begin{array}{c}\otimes \\
\text { (No possible defence) } \\
\end{array}$ \\
\hline
\end{tabular}

The idea for disjunction is that the proposition $A \vee B$, when asserted by a player, is challenged by the question "Which one?"; the defender has then to choose one of the disjuncts and to defend it against any new attack. The rule is the same for the conjunction $A \wedge B$, except that the choice is now made by the attacker: "Give me the left conjunct $\left(?_{L}\right)$ " or "Give me the right one $\left(?_{R}\right)$ ", and the defender has to assume the conjunct chosen by his or her challenger. For the conditional $A \rightarrow B$, the attacker assumes the antecedent $A$ and the defender continues with $B$. Finally negated formulas are attacked by the cancellation of negation, and cannot be defended. The defender in this case can thus only counterattack (if she can).

Structural Rules In addition to the particle rules connected to each logical constant, one also needs structural rules to be able to play in such and such a way at the level of the whole game.

- (PL-0) Starting Rule: The initial formula (the thesis of the dialogical game) is asserted by $\mathbf{P}$. Moves are numbered and alternatively uttered by $\mathbf{P}$ and $\mathbf{O}$. Each move after the initial utterance is either an attack or a defence.

- (PL-1) Winning Rule: Player $\mathbf{X}$ wins iff it is $\mathbf{Y}$ 's turn to play and $\mathbf{Y}$ cannot perform any move.

- (PL-2) No Delaying Tactics Rule: Both players can only perform moves that change the situation. 
- (PL-3) Formal Rule: (In a given context $\mathbf{P}$ ) cannot introduce any new atomic formula; new atomic formulas must be stated by $\mathbf{O}$ first. Atomic formulas can never be attacked.

These four rules are common to dialogical games for both classical and intuitionistic logic. The only difference resides in the following rule:

- (PL-4c) Classical Rule: In any move, each player may attack a complex formula uttered by the other player or defend him/herself against any attack (including those that have already been defended).

- (PL-4i) Intuitionistic Rule: In any move, each player may attack a complex formula uttered by the other player or defend him/herself against the last attack that has not yet been defended.

Now we can build two distinct sets of rules DialPLc and DialPLi, yielding respectively classical propositional logic and intuitionistic propositional logic:

$$
\begin{aligned}
\text { DialPLc }:=\text { PartRules } \cup\{\text { PL-0, PL-1, PL-2, PL-3, PL-4c }\} \\
\text { DialPLi }:=\text { PartRules } \cup\{\text { PL-0, PL-1, PL-2, PL-3, PL-4i }\}
\end{aligned}
$$

This means that the difference between classical and intuitionistic logic is reducible to the structural rule PL-4: all the other structural rules, like particle rules, are strictly identical.

For any set of rules $\Sigma$, I will use the notation $\Sigma \vDash A$ to say that there is a winning strategy for the proponent in the dialogical game about $A$ played according to the rules of $\Sigma$. As PL-4i is more constrained than PL-4c, we have for any propositional formula: $\quad$ DialPLi $\vDash A \Rightarrow$ DialPLc $\vDash A$.

Example 1 As a first example of a dialogical game for propositional logic, let's consider a formula that is valid according to both classical and intuitionistic logic: $((a \rightarrow b) \wedge a) \rightarrow b$. In the dialogical frame, it means that there is a winning strategy for the proponent $\mathbf{P}$ when she plays according to both sets of rules. The rounds and the corresponding arguments, attacks or defences, are indicated by a number within brackets $(n)$ in the external columns, whereas the arguments attacked by the players are referred to by their number $n$ in the internal column. Defences are on the lines of the corresponding attacks. The reader can check the following game and see what is

\begin{tabular}{|c|c|c|c|c|c|}
\hline \multicolumn{3}{|c|}{$\mathrm{O}$} & \multicolumn{3}{|c|}{$\mathbf{P}$} \\
\hline & & & & $((a \rightarrow b) \wedge a) \rightarrow b$ & $(0)$ \\
\hline$(1)$ & $(a \rightarrow b) \wedge a$ & 0 & & $b$ & $(8)$ \\
\hline$(3)$ & $a \rightarrow b$ & & 1 & $? ?_{L}$ & $(2)$ \\
\hline$(5)$ & $a$ & & 1 & $?_{R}$ & $(4)$ \\
\hline$(7)$ & $b$ & & 3 & $a$ & $(6)$ \\
\hline
\end{tabular}
the winning strategy employed by $\mathbf{P}$ :

Having stated the thesis (0), $\mathbf{P}$ cannot simply defend it against $\mathbf{O}$ 's first attack (1) since she should assert $b$ which is an atom not yet stated by the opponent. But $\mathbf{P}$ can

\footnotetext{
${ }^{1}$ In propositional logic contexts are not yet defined - this will be usefull for dialogical games for modal logics.
} 
counterattack twice, with (2) and (4), and $\mathbf{O}$ is forced to defend himself with (3) and (5) respectively. Thanks to (5), $\mathbf{P}$ can use $a$ at round (6) and attack O's round (3) to oblige him to answer $b(7)$. Now $b$ is available to $\mathbf{P}$ who can answer the first attack and win the game (no more move being permitted for the opponent.)

Example 2 The second example is provided by the dialogical games associated to the formula: $\neg \neg a \rightarrow a$. As can be expected, we will get DialPLc $\vDash \neg \neg a \rightarrow a$, but DialPLi $\not \models \neg \neg a \rightarrow a:$

\begin{tabular}{|c|c|c|c|c|c|}
\hline \multicolumn{3}{|c|}{$\mathrm{O}$} & \multicolumn{3}{|c|}{$\mathbf{P}$} \\
\hline & & & & $\neg \neg a \rightarrow a$ & $(0)$ \\
\hline (1) & $\neg \neg a$ & 0 & & $a$ & $(4)$ \\
\hline & $\otimes$ & & 1 & $\neg a$ & $(2)$ \\
\hline (3) & $a$ & 2 & & $\otimes$ & \\
\hline
\end{tabular}

The difference between the games happens after round (3). Following the intuitionistic rule (PL-4i), the proponent should defend herself against the last attack not yet defended, i.e. against (3); but she cannot, since (3) is an attack against a negation leaving no available defence. By contrast, according to the classical rule, the proponent can defend herself against a former attack of the opponent, so she can answer (4) to (1), and win the game.

\section{2 (Modal) Epistemic logic}

As for PL, Modal Logic requires the introduction of particle and structural rules corresponding to the additional operators. We will moreover need a convention to designate the different contexts (or possible worlds) where propositions are stated by both players.

Particle Rules The thesis of the dialogue is uttered in a given context $w$. The particle rules for the epistemic operator $K$ and for its dual $P$ enable the players to change the context.

\begin{tabular}{|c|c|c|}
\hline & Attack & Defence \\
\hline$K A$ & $?_{K / w^{\prime}}$ & $A$ \\
$($ in context $w)$ & $\begin{array}{c}(\text { The attacker chooses } \\
\left.\text { an available context } w^{\prime}\right)\end{array}$ & $\left(\right.$ in context $\left.w^{\prime}\right)$ \\
\hline$P A$ & $?_{P}$ & $\begin{array}{c}A \\
\text { (in an available context } w^{\prime} \\
\text { chosen by the defender) }\end{array}$ \\
\hline
\end{tabular}

\section{Contexts numbering}

- The initial context is numbered 1 . The $n$ immediate successors of $m$ are numbered $m .1, m .2, \ldots, m . n$.

- An immediate successor $m . n$ of a context $m$ is said to be of rank +1 relative to $m$, and $m$ is said to be of rank -1 relative to its immediate successors. A successor m.n.p of a context $m$ is said to be of rank +2 relative to $m$, etc. 
Structural Rules Modal structural rules correspond to restrictions on the accessibility relation $\mathcal{K}$ between contexts (and thus determine which contexts are available to players). The first two rules are obviously incompatible and should not be included together in the same set of rules.2

- (ML-frc) Formal Rule for Contexts: P cannot introduce a new context; new contexts must be introduced by $\mathbf{O}$ first.

- (ML-D) Axiom D rule: $\mathbf{P}$ can introduce a new context of rank +1 relative to the context he is playing in.

- (ML-K) K Rule: $\mathbf{P}$ cannot stay in the context she is playing in (as she attacks a formula of the form $K A$ or defends a formula of the form $P A$ ). $\mathbf{P}$ can choose a (given) context of rank +1 relative to the context she is playing in.

- (ML-T) T Rule: $\mathbf{P}$ can either choose a (given) context of rank +1 relative to the context she is playing in, or stay in the context she is playing in.

- (ML-B) B Rule: $\mathbf{P}$ can either choose a (given) context of rank $-1 /+1$ relative to the context she is playing in, or stay in the context she is playing in.

- (ML-S4) S4 Rule: $\mathbf{P}$ can either choose any (given) context of rank $+k$ relative to the context she is playing in, or stay in the context she is playing in.

- (ML-S5) S5 Rule: P can choose any (given) context.

Dialogical Epistemic Systems Combining these new rules with those of DialPLc, one obtains sets of rules corresponding to different usual systems of propositional modal logic:

$$
\begin{aligned}
\text { DialK }:=\text { DialPLc } \cup\{\text { ML-frc, ML-K }\} \\
\text { DialD }:=\text { DialPLc } \cup\{\text { ML-D, ML-K }\} \\
\text { DialT }:=\text { DialPLc } \cup\{\text { ML-frc, ML-T }\} \\
\text { DialB }:=\text { DialPLc } \cup\{\text { ML-frc, ML-B }\} \\
\text { DialS4 }:=\text { DialPLc } \cup\{\text { ML-frc, ML-S4 }\} \\
\text { DialS5 }:=\text { DialPLc } \cup\{\text { ML-frc, ML-S5 }\}
\end{aligned}
$$

Example Let's consider a substitution instance of the Positive Introspection Property (also known as Axiom 4): $K \phi \rightarrow K K \phi$, to be played according to DialS4. What is interesting here is the fact that the proponent resorts to the transitivity of $\mathcal{K}$ at round $(6)$ - if the game was played in a non-transitive structure, there would be no more winning strategy available to $\mathbf{P}$.

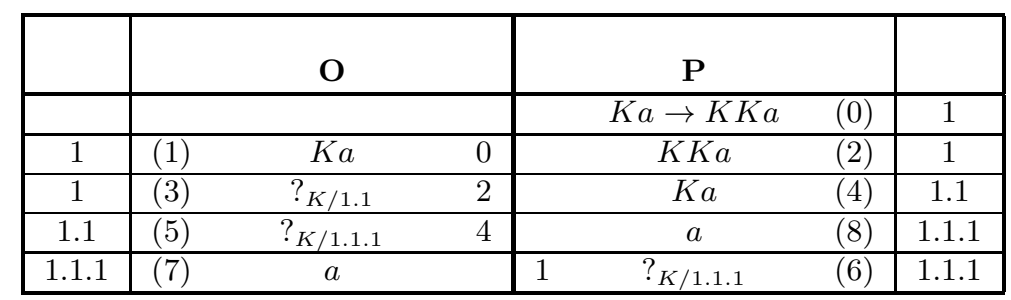

\footnotetext{
${ }^{2}$ Here I follow Rahman and Rückert's formulation of rules associated with specific modal systems. Structural rules could also be formulated in accordance with the specific axioms involved in those systems: the upshot would be the same.
} 


\section{Intuitionistic DEL}

Two kinds of Intuitionistic epistemic logic can be provided using the dialogical frame. The first one is Intuitionistic Modal Logic to be sketched in this section. The second one is a dialogical version of the modal simulation of intuitionistic logic, to be presented in the next section.

\subsection{Intuitionistic Modal Logic}

Within the Dialogical frame, Rahman \& Rückert [12] suggest just to change DialPLc into DialPLi in the set of structural rules. For instance, an intuitionistic version of S5 is directly obtained by replacing (PL-4c) by (PL-4i): DialS5i := DialPLi $\cup\{$ MLfrc, ML-S5\}. For any dialogical system of modal logic Dial $\Sigma$, I will use the notation $\operatorname{Dial} \Sigma \mathbf{i}$ to designate the corresponding intuitionistic version obtained in this way.

Is it really intuitionistic modal logic? Such dialogical systems are obtained through a simple combinatorial step. It can be doubted that they yield "real" intuitionistic modal logics. Let $\mathcal{L}_{M}$ be the standard propositional language augmented by the modal connectives in $M$. Wolter \& Zakharyaschev's general definition of an intuitionistic modal logic $\mathcal{L}$ in $\mathcal{L}_{M}$ is as follows 114: (1) $\mathcal{L} \subset \mathcal{L}_{M} ;(2) \mathcal{L}$ contains propositional intuitionistic logic; (3) $\mathcal{L}$ is closed under: (i) Modus ponens, (ii) Substitution, (iii) Regularity Rule $(A \rightarrow B / \bigcirc A \rightarrow \bigcirc B$, for every $\bigcirc \in M$.)

In the dialogical frame, it is easily seen that conditions (1) and (2) are automatically filled with the relations holding between the corresponding sets of rules Dial $\boldsymbol{\Sigma}$ and $\mathbf{D i a l} \boldsymbol{\Sigma} \mathbf{i}$. Now, one can easily check that any dialogical epistemic system $\mathbf{D i a l} \boldsymbol{\Sigma} \mathbf{i}$ is closed under the Regularity Rule:

\begin{tabular}{|c|c|c|c|c|c|c|}
\hline & \multicolumn{3}{|c|}{$\mathrm{O}$} & \multicolumn{2}{|l|}{$\mathbf{P}$} & \\
\hline & & & & $A \rightarrow B$ & $(0)$ & 1 \\
\hline 1 & (1) & $\bar{A}$ & 0 & $B$ & $(2)$ & 1 \\
\hline & & ... & & $\ldots$ & & \\
\hline
\end{tabular}

$A \rightarrow B$ is valid iff there is a winning strategy for $\mathbf{P}$ to end this game.

\begin{tabular}{|c|c|c|c|c|c|c|c|}
\hline & \multicolumn{3}{|c|}{$\mathrm{O}$} & & \multicolumn{2}{|l|}{$\mathbf{P}$} & \\
\hline & & & & & $K A \rightarrow K B$ & $\overline{(0)}$ & 1 \\
\hline$\overline{1}$ & $(1)$ & $K A$ & $\overline{0}$ & & $K B$ & $(2)$ & 1 \\
\hline 1 & (3) & $?_{K / 1.1}$ & 2 & & $B$ & (6) & 1.1 \\
\hline 1.1 & (5) & $A$ & & 1 & $?_{K / 1.1}$ & (4) & 1.1 \\
\hline & & $\ldots$ & & & $\ldots$ & & \\
\hline
\end{tabular}

\begin{tabular}{|c|c|c|c|c|c|c|c|}
\hline & \multicolumn{3}{|c|}{$\mathrm{O}$} & \multicolumn{3}{|c|}{$\mathbf{P}$} & \\
\hline & & & & & $P A \rightarrow P B$ & $(0)$ & 1 \\
\hline 1 & $\overline{(1)}$ & $P A$ & 0 & & $P B$ & $(2)$ & 1 \\
\hline 1 & (3) & $?_{P}$ & 2 & & $B$ & $(6)$ & 1.1 \\
\hline 1.1 & (5) & $\bar{A}$ & & 1 & $?_{P}$ & (4) & 1 \\
\hline & & $\ldots$ & & & $\ldots$ & & \\
\hline
\end{tabular}


What follows immediately from the last two dialogues is that they are to be continued in the same way as that corresponding to $A \rightarrow B$. In other words: If there is a winning strategy for $\mathbf{P}$ in the dialogue associated to $A \rightarrow B$, then there is one for the corresponding dialogue associated to $K A \rightarrow K B$ (or $P A \rightarrow P B$ ).

Moreoever, the dialogical system DialKi at least encompasses Fischer-Servi's intuitionistic modal logic FS, and the same for the corresponding extensions S4, S5, and so forth. (For more details, see the Appendix.)

\subsection{Application to epistemic modalities}

Knowledge Generalization The necessitation rule KG (i.e. $A / K A$ ), applies only to intuitionistic validities, not to classical ones:

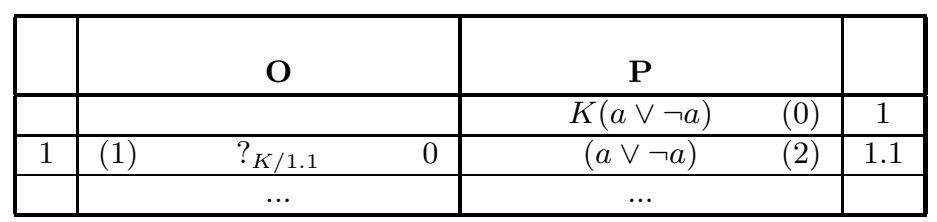

After (2), the play goes on (in context 1.1) according to DialPLi. Hence O wins! Intuitionistic dialogical epistemic systems thus account for (explicit) knowledge of intuitionist agents.

Intuitionistic $\mathbf{K}$ and $\mathbf{P}$ In intuitionistic modal systems Dial $\mathbf{\Sigma i}, K$ and $P$ become genuine intuitionistic modal operators: they are no more interdefinable.

For instance according to DialTi (i.e. with a reflexive accessibility relation), one can see that: $\neg K \neg A \not ̋ P A$ - the following dialogue stops at round (7) -, whereas (as is expected): $\neg K \neg A \approx P A$ within DialT - the play goes on.

\begin{tabular}{|c|c|c|c|c|c|c|c|}
\hline & \multicolumn{3}{|c|}{$\mathrm{O}$} & \multicolumn{3}{|c|}{$\mathbf{P}$} & \\
\hline & & & & & $\neg K a \rightarrow P \neg a$ & $(0)$ & 1 \\
\hline 1 & (1) & $\neg K a$ & 0 & & $P \neg a$ & $(2)$ & 1 \\
\hline 1 & (3) & $?_{P}$ & 2 & & $\neg a$ & $(4)$ & 1 \\
\hline 1 & $(5)$ & $a$ & 4 & & $\otimes$ & & \\
\hline & & $\otimes$ & & 1 & $K a$ & $\overline{(6)}$ & 1 \\
\hline 1 & $(7)$ & $?_{K / 1.1}$ & 6 & & $a$ & $(\mathbf{1 0})$ & 1.1 \\
\hline & $\left(3^{\prime}\right)$ & $?_{P}$ & 2 & & $\neg a$ & $(8)$ & 1.1 \\
\hline 1.1 & $(\mathbf{9})$ & $a$ & 8 & & $\otimes$ & & \\
\hline
\end{tabular}

It also can be shown that other properties of $K$ and $P$ still hold in DialTi, such as the Consistency Property (D) $\mathrm{H}^{3}$

\footnotetext{
${ }^{3}$ The Consistency Property is not valid according to DialKi, as can be seen at round (4): with no reflexive accessibility relation, $\mathbf{P}$ cannot choose the current context to attack O's assertion of $K a$.
} 


\begin{tabular}{|c|c|c|c|c|c|c|c|}
\hline & \multicolumn{3}{|c|}{$\mathbf{O}$} & & \multicolumn{2}{|l|}{$\mathbf{P}$} & \\
\hline & & & & & $\overline{K a} \rightarrow P a$ & (0) & 1 \\
\hline 1 & (1) & $K a$ & 0 & & $P a$ & $(2)$ & 1 \\
\hline 1 & (3) & $?_{P}$ & 2 & & $\bar{a}$ & (6) & 1 \\
\hline 1 & (5) & $a$ & & 1 & $?_{K / 1}$ & (4) & 1 \\
\hline
\end{tabular}

Advantages of Intuitionistic DEL To conclude this section, let's mention a few features of Intuitionistic DEL which make it a good tool for epistemic logic:

1. Intuitionistic DEL provides an interesting account of modalities $K$ and $P$ : Ignoring $a$ does no longer imply considering $\neg a$ as a possibility.

2. Implicit epistemic logic is made explicit: the intuitionist agent is described as an intuitionist agent (thanks to the aforementioned restriction of KG to intuitionistic valid formulas). One could change the rules of the underlying propositional logic (e.g. for more strictly contructive ones) and obtain a corresponding explicit epistemic version in the same straighforward manner.

3. With the intuitionistic operators $K$ and $P$, not only the described agent but his/her interpreter too is (implicitly) grasped as a cognitive agent. This may be illustrated by the rejection of the tertium non datur: $K A \vee \neg K A$, in DialS5i:

\begin{tabular}{|c|ccc|cc|c|}
\hline & \multicolumn{3}{|c|}{$\mathbf{O}$} & \multicolumn{2}{|c|}{$\mathbf{P}$} & \\
\hline & & & & $K a \vee \neg K a$ & $(0)$ & 1 \\
\hline 1 & $(1)$ & $?$ & 0 & $\neg K a$ & $(2)$ & 1 \\
\hline 1 & $(3)$ & $K a$ & 2 & $?_{K / 1}$ & $(4)$ & 1 \\
\hline 1 & $(5)$ & $a$ & & 3 & $?^{2}$ & \\
\hline
\end{tabular}

\section{Modal Simulation of Intuitionistic (non-modal) Logic}

Let's turn again to implicit epistemic logic, namely intuitionistic propositional logic Int. In the first section, I presented the system DialPLi which is the usual dialogical implementation of Int. In this section, I will propose a new dialogical formulation of intuitionistic logic, grounded on Gödel's 1933 S4 embedding of Int, and on Kripke's 1965 modal semantics for Int.

Such a formulation is based on a dynamic conception of knowledge: The accessibility relation between contexts corresponds to tense and to the growth of information - in contrast to Hintikka's 1962 "static" conception of this relation.

\subsection{Gödel's embedding and Kripke's semantics}

Gödel's translation of Int into S4 The idea of Gödel's embedding is closely related to the BHK interpretation of intuitionistic logic. When a formula is known (one could say: 'proved'), it will persist through time. The underlying idea is that of an always increasing knowledge, with neither memory failure nor revision. Formally, the $\mathbf{S} 4$-translation $A^{T}$ of an intuitionistic formula $A$ is as follows: 


$$
\begin{aligned}
& a^{T}:= \square a \text { for every atomic formula } a \\
&(A \wedge B)^{T}:=A^{T} \wedge B^{T} \\
&(A \vee B)^{T}:=A^{T} \vee B^{T} \\
&(\neg A)^{T}:=\square \neg A^{T} \\
&(A \rightarrow B)^{T}:=\square\left(A^{T} \rightarrow B^{T}\right)
\end{aligned}
$$

It leads to the expected equivalence: $\quad \vDash_{\text {Int }} A$ iff $\vDash_{\mathbf{S} 4} A^{T}$.

Kripke's modal semantics for Int Kripke's [8] structures involve a reflexive and transitive relation $\leq$ between contexts. The idea is similar to Gödel's translation: there is a temporal ordering of worlds, propositions being established once for all if they are, and the same for negated propositions. So before being known, a proposition is not true and neither is its negation.

Formally, a Kripke structure is thus a tuple $\mathcal{K}=\langle W, \leq, \Vdash\rangle$, where: $(1) \leq$ is a pre-ordering on $W$ (i.e. a binary reflexive and transitive relation); (2) the forcing relation $\Vdash$ is such that: (2.1) For all $w \in W, w \| \perp(2.2)$ For all $w, w^{\prime} \in W$, if $w \leq w^{\prime}$ and $w \Vdash a$, then $w^{\prime} \Vdash a$ (where $a$ is an atomic formula). The forcing relation is then extended to complex formulas according to the following requirements: $(i) w \Vdash A \wedge B$ iff $w \Vdash A$ and $w \Vdash B$; $(i i) w \Vdash A \vee B$ iff $w \Vdash A$ or $w \Vdash B$; (iii) $w \Vdash A \rightarrow B$ iff $\forall w^{\prime} \in W$, if $w \leq w^{\prime}$ and $w^{\prime} \Vdash A$, then $w^{\prime} \Vdash B$; (iv) $w \Vdash \neg A$ iff $\forall w^{\prime} \in W$, if $w \leq w^{\prime}$ then $w^{\prime} \nVdash A$.

Now we have the following equivalence:

$\vDash_{\text {Int }} A$ iff $\mathcal{K} \Vdash A$ for any Kripke model $\mathcal{K}$.

\subsection{Dialogical Simulation of Int}

Can the modal simulation of Int be implemented in dialogical games? The idea is to consider propositional intuitionistic formulas as if they were modal formulas. A dialogical version of $\mathbf{S} \mathbf{4}$ obtains thanks to the usual structural rules. Of course, there will be a restriction on the formulas: we reach a $\mathbf{S 4}$-like dialogical version for the propositional fragment only (i.e. not for formulas including modal operators).

However, the usual S4 structural rules are not enough for this implementation: we must take into account the non-standard interpretation of atoms, negation and subjunction. Eventually, our system $\mathbf{I n t}^{\mathbf{S} 4}$ will essentially differ from $\mathbf{S} 4$ at the level of particle rules.

Atoms According to Gödel's translation: $a^{T}:=\square a$ for every atomic formula $a$. Let's consider the following part of game involving $\square a$ and played with DialS4:

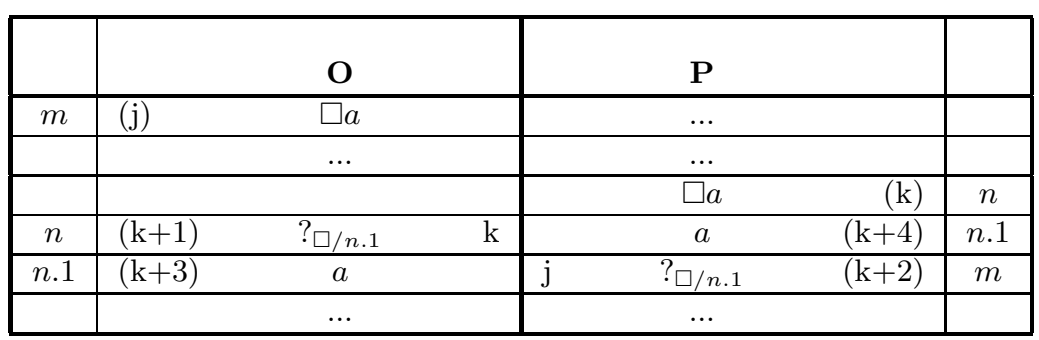

Player $\mathbf{P}$ can defend her assertion of $\square a$ only if $\square a$ has been previously introduced by $\mathbf{O}$ in any context $m \leq n$ (thanks to the transitivity of $\leq$ in $\mathbf{S 4}$ ). So (S4 translations 
of) Int atoms can be attacked since they are modal formulas. We should thus add a special particle rule for atoms in $\mathbf{I n t}^{\mathbf{S 4}}$, stating that if an atom $a$ is asserted in a context $m$, then it can be attacked by $?_{n}$ where the attacker chooses an available context $n \geq m$, and defended by the assertion of $a$ in the context $n$. Correlatively, the formal rule (PL-3) should be modified to enable players to attack atomic formulas. We would get the following structural rule.

But we do not need to change the particle rule for atomic formula. A simple look at the situation makes it clear that the whole modification can be restricted to one structural rule:

- $\left(\mathrm{PL}-3^{*}\right)$ Int $^{\mathbf{S} 4}$ Formal Rule: In a given context $n \mathbf{P}$ cannot introduce any new atomic formula that has not been introduced by $\mathbf{O}$ in any context $m \leq n$; new atomic formulas must be stated by $\mathbf{O}$ first. Atomic formulas can never be attacked.

Negation Gödel's translation $(\neg A)^{T}:=\square \neg A^{T}$ indicates that a negated formula asserted in a context $m$ can be challenged in any context $n \geq m$ :

\begin{tabular}{|c|c|c|c|c|c|}
\hline & \multicolumn{2}{|r|}{$\mathrm{O}$} & $\mathbf{P}$ & & \\
\hline & \multicolumn{2}{|r|}{$\ldots$} & $\ldots$ & & \\
\hline & & & $\square \neg A$ & $(\mathrm{k})$ & $n$ \\
\hline$n$ & $(\mathrm{k}+1)$ & $? \square / n .1$ & $\neg A$ & $(\mathrm{k}+2)$ & $n .1$ \\
\hline$n .1$ & $(\mathrm{k}+3)$ & $\begin{array}{ll}\mathrm{k}+2 \\
\end{array}$ & $\otimes$ & & \\
\hline & & $\ldots$ & $\ldots$ & & \\
\hline
\end{tabular}

This leads naturally to the following Particle rule for negation in $\mathbf{I n t}^{\mathbf{S 4}}$ :

\begin{tabular}{|c|c|c|}
\hline & Attack & Defence \\
\hline$\neg A$ & $A$ & $\otimes$ \\
$($ in context $m)$ & $\begin{array}{c}\text { (in an available context } n \geq m \\
\text { chosen by the attacker) }\end{array}$ & \\
\hline
\end{tabular}

Implication The case of implication $(A \rightarrow B)^{T}:=\square\left(A^{T} \rightarrow B^{T}\right)$ is similar to that of negation:

\begin{tabular}{|c|c|c|c|c|c|c|}
\hline & \multicolumn{3}{|c|}{$\mathrm{O}$} & \multicolumn{2}{|l|}{$\mathbf{P}$} & \\
\hline & & & & $\ldots$ & & \\
\hline & & & & $\square(A \rightarrow B)$ & $(\mathrm{k})$ & $n$ \\
\hline$n$ & $(\mathrm{k}+1)$ & $? \square / n .1$ & $\mathrm{k}$ & $A \rightarrow B$ & $(\mathrm{k}+2)$ & $\overline{n .1}$ \\
\hline$n .1$ & $(\mathrm{k}+3)$ & $A$ & $\mathrm{k}+2$ & $B$ & $(\mathrm{k}+4)$ & $n .1$ \\
\hline & & $\ldots$ & & $\ldots$ & & \\
\hline
\end{tabular}

It leads to the following modified Particle rule in $\mathbf{I n t}^{\mathrm{S} 4}$ :

\begin{tabular}{|c|c|c|}
\hline & Attack & Defence \\
\hline $\begin{array}{c}A \rightarrow B \\
\text { (in context } m \text { ) }\end{array}$ & $\begin{array}{c}A \\
\text { (The attacker chooses } \\
\text { an available context } n \geq m)\end{array}$ & $\begin{array}{c}B \\
\text { (in context } n \text { ) }\end{array}$ \\
\hline
\end{tabular}


Recapitulation To sum up our new system, let's denote the new set of particle rules by PartRules ${ }^{\mathbf{S} 4}$ : it is thus identical to PartRules concerning conjunction and disjunction, and differs on subjunction and negation.

Now we get a new set of rules:

$$
\text { Int }^{\mathrm{S} 4}:=\text { PartRules }^{\mathrm{S} 4} \cup\left\{\mathrm{PL}-0, \mathrm{PL}-1, \mathrm{PL}-2, \mathrm{PL}-3^{*}, \mathrm{PL}-4 \mathrm{c}\right\} \cup\{\mathrm{ML}-\mathrm{frc}, \mathrm{ML}-\mathrm{S} 4\}
$$

which is equivalent to DialPLi in the following sense:

$$
\text { Int }^{\mathrm{S} 4} \vDash A \Leftrightarrow \mathbf{D i a l P L i} \vDash A
$$

for any propositional formula $A$.

Example $1 \quad$ Int $^{\text {S4 }} \not \models a \vee \neg a$

\begin{tabular}{|c|ccc|cc|c|}
\hline & \multicolumn{2}{|c|}{$\mathbf{O}$} & \multicolumn{2}{|c|}{$\mathbf{P}$} & \\
\hline & & & & $a \vee \neg a$ & $(0)$ & 1 \\
\hline 1 & $(1)$ & $?$ & 0 & $\neg a$ & $(2)$ & 1 \\
\hline 1.1 & $(3)$ & $a$ & 2 & $\otimes$ & & \\
\hline
\end{tabular}

\begin{tabular}{|c|c|c|c|c|c|c|c|}
\hline & \multicolumn{3}{|c|}{ O } & \multicolumn{3}{|c|}{$\mathbf{P}$} & \\
\hline & & & & & $\neg \neg(a \vee \neg a)$ & $\overline{(0)}$ & 1 \\
\hline 1.1 & (1) & $\neg(a \vee \neg a)$ & 0 & & $\otimes$ & & \\
\hline & & $\otimes$ & & 1 & $a \vee \neg a$ & $\overline{(2)}$ & 1.1 \\
\hline 1.1 & $\overline{(3)}$ & $?$ & 2 & & $\neg a$ & (4) & 1.1 \\
\hline 1.1 .1 & (5) & $a$ & 4 & & $\otimes$ & & \\
\hline & & $\otimes$ & & 1 & $a \vee \neg a$ & $\overline{(6)}$ & 1.1 .1 \\
\hline 1.1 .1 & (7) & $?$ & 6 & & $a$ & $(8)$ & 1.1 .1 \\
\hline
\end{tabular}

Example 2 Int $^{\mathrm{S} 4} \vDash \neg \neg(a \vee \neg a)$

\section{Discussion}

In Game-Theoretical Semantics (GTS), one can distinguish between two types of "knowledge" depending on whether one is concerned about the interpretation of the epistemic operators (i.e. the usual meaning of "knowledge") or about the knowledge of the players of evaluation games. Van Benthem [2] strongly stresses the epistemic features involved in GTS and IF Logic, even though such an interpretation of imperfect information games is absent from Hintikka's original creation.

A similar distinction can be made in the dialogical frame, between explicit knowledge (that is embedded in the operators) and implicit knowledge (of the players). However, the distinction is not exactly the same since the players of dialogical games are not assumed to have particular information sets at their diposal, but a set of action rules. The intuitionistic restriction (PL-4i) imposed on the set of classical rules for PL therefore leads to a modelling of an abstract agent with limited (i.e. intuitionistic) epistemic powers.

In the above sections, two competing accounts of knowledge using dialogical games are provided. The two implementations resort to both implicit and explicit knowledge. 
They consist in specific combinations of intuitionistic and modal logics. Whereas systems Dial $\Sigma \mathbf{i}$ innoculate an intuitionistic variation to standard dialogical systems of modal logic, $\mathbf{I n t}^{\mathrm{S} 4}$ (implicitely) involves a modal interpretation of intuitionistic logic.

The two approaches presented in this paper could be extended in several ways. Among the possible developments of what should be called in general Dialogical Epistemic Logic, we highlight the following:

1. As was already stressed, $\mathbf{I n t}^{\mathrm{S} 4}$ is only concerned with the propositional fragment of S4. One could easily consider modal extensions $\mathbf{I n t}_{\mathbf{K}}^{\mathbf{S} 4}$ of it, using the underlying Kripke-like modal structure, and consider assertions about the S4knowledge of an intuitionistic agent, such as e.g.: $\neg K(a \vee \neg a)$.

2. Dialogical systems of doxastic logic, e.g. the system DialKD45. Doxastic logic in the dialogical frame starts like a nice story. The structural rule (ML-D) which separates the logic of belief from the logic of knowledge specifically enables the proponent to create new contexts... In doxastic logic too, intuitionistic variations could be easily implemented.

3. Multi-agent epistemic logic: such a development would be naturally grou-nded in multi-modal dialogical logic.

4. Non-Normal Logics. These "deviant" modal logics are due to Lemmon 1957 and Kripke 1965. They are based on the rejection of the axiom $\mathbf{K}$ and/or of the Necessitation Rule (KG in epistemic logic: $A / K A$ ). A motivation for adopting such a logic is to escape logical omniscience. Several authors have supplied epistemic logics using non-normal logic:

- Duc [f] uses normal action modalities and non-normal epistemic modalities, where $\mathbf{K G}$ is replaced by: $A /\left\langle F_{i}\right\rangle K A,\left\langle F_{i}\right\rangle$ being a dynamic actiontemporal modality;

- Thomason's theory 13] is a combination of a normal (KD45) multi-agent frame and of a non-normal (E2) intra-agent frame (between subagents).

Rahman and Keiff's recent proposal of a dialogical implementation of nonnormal logics ([10], 11] ) leads to an immediate epistemic interpretation. Their main idea consists in considering a kind of meta-modal logic, i.e. a frame in which it is possible to consider different modal systems together. For example, an intuitionist logician might want to consider the (possible) case where tertium non datur were valid in his or her logic: the case in question amounts to a context where another logic is assumed to hold. Transposing it to epistemic logic enables one to consider the following cases:

- Standard interpretation of a deviant agent in multi-modal epistemic logic, e.g. $K_{i} K_{j} A$ where agent the $j$ is crazy and the agent $i$ is sane;

- Deviant interpretations of standard epistemic agents;

- Shifting positive introspection (this is made by a crazy agent who knows he or she is crazy, like in $K_{i}^{\text {sane }} K_{i}^{\text {crazy }} A$.)

Let's give a quick illustration of this non-normal frame with the dialogical game of the formula $K^{c} K^{i}(a \vee \neg a)$ - stating that one knows classically that she knows intuitionistically that $a$ or not $a$, which is surely false. This is established according to S0.5: 


\begin{tabular}{|c|ccc|cc|c|}
\hline & \multicolumn{3}{|c|}{$\mathbf{O}$} & & $\mathbf{P}$ & \\
\hline & & & & $K K(a \vee \neg a)$ & $(0)$ & $1-\mathrm{PLc}$ \\
\hline $1-\mathrm{PLc}$ & $(1)$ & $?_{K / 1.1}$ & 0 & $K(a \vee \neg a)$ & $(2)$ & $1.1-\mathrm{PLc}$ \\
\hline $1.1-\mathrm{PLc}$ & $(3)$ & $?_{K / 1.1 .1-P L i}$ & 2 & $a \vee \neg a$ & $(4)$ & $1.1 .1-\mathrm{PLi}$ \\
\hline $1.1 .1-\mathrm{PLi}$ & $(5)$ & $?$ & 4 & $\neg a$ & $(6)$ & $1.1 .1-\mathrm{PLi}$ \\
\hline $1.1 .1-\mathrm{PLi}$ & $(7)$ & $a$ & 6 & $\otimes$ & & \\
\hline
\end{tabular}

(An underlying logic, here DialPLc or DialPLi, is associated to each context. For explanations of such dialogues, see the quoted papers.)

\section{Conclusion}

The original formulation of dialogical logic by Lorenzen and Lorenz [9] was strongly related to intuitionistic logic. However, this connection may be enriched as is shown by our implementation of Gödel's S4 embedding of Int. With the system Int ${ }^{\text {S4 }}$ one gets a new version of intuitionistic logic where the interpretation is directly linked to the connectives, at the level of the particle rules - like in the BHK interpretation.

In this paper I have sketched two systems of epistemic logic, conceptually very different but implementationally very close. While presenting systems $\mathbf{D i a l} \mathbf{\Sigma i}$, we have seen that genuine intuitionistic modal logics resulted easily. However, lots of perspectives in the field of dialogical epistemic logic go far beyond the scope of this paper, as for instance the exact delimitation of such systems, extensions to multi-modal logics or to non-normal systems.

A new insight on the relationship between intuitionistic and modal logics has been provided by dialogical logic. It is at least a confirmation of the fecondity of dialogical logic as a frame to compare logical theories.

\section{Appendix: Dialogical games and intuitionistic ML}

DialKi and (FS) DialKi appears to be at least equivalent to the well-known system of intuitionistic modal logic due to Fischer-Servi (FS): the axioms of (FS) are all valid according to the dialogical set of rules DialKi. This is shown through dialogical games; a real proof would require a demonstration exhibiting every possible strategy for the opponent - this can be accomplished with Beth-Smullyan-like tableaux, where formulas are prefixed with the name of the player, $\mathbf{P}$ or $\mathbf{O}$. (See [6] and [12] for details.)

The set of axioms of FS is the union of $\mathbf{I n t K}_{\square}$ (the extention of propositional Int with the standard modal axioms $\mathrm{K}$ for $\square$ ), $\mathbf{I n t K}_{\diamond}$ (the same except that the standard modal axioms $K$ are given for $\diamond$ ), and two specific axioms (see [3], 14]). We will need to add the symbol $\perp$ as a prime formula, and define $\neg$ and $\top$ with it. Particle rules for $\perp$ and $T$ immediately follow from these definitions:

- $\neg A:=A \rightarrow \perp$, so $\perp$ can never be stated (if it could, negation would be defensible);

- $\top:=\neg \perp$, so $\top$ can be stated by any player in any context (it is not an atomic formula) and it cannot be attacked. 
In what follows, the games corresponding to each axiom are written down without comment. Every game is won by player $\mathbf{P}$, according to some winning stragegy. So for every axiom $A$ of $\mathbf{F S}$, we get: $\quad$ DialKi $\vDash A$.

IntK $\square$ : Int, $\square \top, \square(a \wedge b) \leftrightarrow(\square a \wedge \square b)$.

\begin{tabular}{|c|c|c|c|c|c|c|}
\hline & \multicolumn{3}{|c|}{$\mathrm{O}$} & \multicolumn{2}{|l|}{$\mathbf{P}$} & \\
\hline & & & & $\square T$ & $(0)$ & 1 \\
\hline 1 & $\overline{(1)}$ & $? \square / 1.1$ & 0 & $\bar{T}$ & (2) & 1.1 \\
\hline
\end{tabular}

\begin{tabular}{|c|c|c|c|c|c|c|c|}
\hline & \multicolumn{3}{|c|}{$\mathbf{O}$} & & \multicolumn{2}{|l|}{$\mathbf{P}$} & \\
\hline & & & & & $\square(a \wedge b) \rightarrow(\square a \wedge \square b)$ & $(0)$ & 1 \\
\hline 1 & $(1)$ & $\overline{\square(a \wedge b)}$ & $\overline{0}$ & & $\square a \wedge \square b$ & $\overline{(2)}$ & 1 \\
\hline 1 & $(3)$ & $\overline{?_{L}}$ & 2 & & $\square a$ & $(4)$ & 1 \\
\hline 1 & $(5)$ & $\begin{array}{l}? \square / 1.1 \\
\end{array}$ & 4 & & $a$ & $(10)$ & 1.1 \\
\hline 1.1 & (7) & $a \wedge b$ & & 1 & $?+\square / 1.1$ & $(6)$ & 1 \\
\hline 1.1 & $(9)$ & $a$ & & 7 & $?_{L}$ & $(8)$ & 1.1 \\
\hline
\end{tabular}

\begin{tabular}{|c|c|c|c|c|c|c|c|}
\hline & \multicolumn{3}{|c|}{$\mathrm{O}$} & \multicolumn{3}{|c|}{$\mathbf{P}$} & \\
\hline & & & & & $(\square a \wedge \square b) \rightarrow \square(a \wedge b)$ & $(0)$ & 1 \\
\hline 1 & (1) & $\square a \wedge \square b$ & $\overline{0}$ & & $\square(a \wedge b)$ & $(2)$ & 1 \\
\hline 1 & $(3)$ & $? \square / 1.1$ & $\overline{2}$ & & $a \wedge b$ & $(4)$ & 1.1 \\
\hline 1.1 & $(5)$ & $?_{L}$ & 4 & & $a$ & $(10)$ & 1.1 \\
\hline 1 & (7) & $\square a$ & & 1 & $?_{L}$ & $(6)$ & 1 \\
\hline 1.1 & (9) & $a$ & & 7 & $? \square / 1.1$ & $(8)$ & 1 \\
\hline
\end{tabular}

IntK$_{\diamond}:$ Int, $\neg \diamond \perp, \diamond(a \vee b) \leftrightarrow(\diamond a \vee \diamond b)$

\begin{tabular}{|c|c|c|c|c|c|c|c|}
\hline & \multicolumn{3}{|c|}{ O } & \multicolumn{3}{|c|}{$\mathbf{P}$} & \\
\hline & & & & & $\neg \diamond \perp$ & $(0)$ & 1 \\
\hline 1 & (1) & $\diamond \perp$ & 0 & & $\otimes$ & & \\
\hline & & $\otimes$ & & 1 & $? \diamond$ & $(2)$ & 1 \\
\hline
\end{tabular}

\begin{tabular}{|c|c|c|c|c|c|c|c|}
\hline & \multicolumn{3}{|c|}{$\mathrm{O}$} & \multicolumn{3}{|c|}{$\mathbf{P}$} & \\
\hline & & & & & $\nabla(a \vee b) \rightarrow(\diamond a \vee \diamond b)$ & $\overline{(0)}$ & 1 \\
\hline 1 & $(1)$ & $\diamond(a \vee b)$ & 0 & & $\diamond a \vee \diamond b$ & (2) & 1 \\
\hline 1 & $(3)$ & $?$ & 2 & & $\nabla a$ & $(8)$ & 1 \\
\hline 1.1 & $(5)$ & $a \vee b$ & & 1 & $?_{\diamond}$ & (4) & 1 \\
\hline 1.1 & $(7)$ & $\bar{a}$ & & 5 & $?$ & $(6)$ & 1.1 \\
\hline 1 & $(9)$ & $? \diamond$ & 8 & & $a$ & $(10)$ & 1.1 \\
\hline
\end{tabular}




\begin{tabular}{|c|c|c|c|c|c|c|c|}
\hline & \multicolumn{3}{|c|}{$\mathrm{O}$} & \multicolumn{3}{|c|}{$\mathbf{P}$} & \\
\hline & & & & & $(\diamond a \vee \diamond b) \rightarrow \diamond(a \vee b)$ & $(0)$ & 1 \\
\hline 1 & $(1)$ & $\diamond a \vee \diamond b$ & 0 & & $\diamond(a \vee b)$ & (2) & 1 \\
\hline 1 & $(3)$ & $?_{\diamond}$ & 2 & & $a \vee b$ & $(8)$ & 1.1 \\
\hline 1 & (5) & $\nabla a$ & & 1 & $?$ & (4) & 1 \\
\hline 1.1 & $(7)$ & $a$ & & 5 & $? \diamond$ & (6) & 1 \\
\hline 1.1 & (9) & $?$ & 8 & & $a$ & (10) & 1.1 \\
\hline
\end{tabular}

FS specific axioms: $\diamond(a \rightarrow b) \rightarrow(\square a \rightarrow \diamond b),(\diamond a \rightarrow \square b) \rightarrow \square(a \rightarrow b)$.

\begin{tabular}{|c|c|c|c|c|c|c|c|}
\hline & \multicolumn{3}{|c|}{$\mathrm{O}$} & \multicolumn{3}{|c|}{$\mathbf{P}$} & \\
\hline & & & & & $\diamond(a \rightarrow b) \rightarrow(\square a \rightarrow \diamond b)$ & $(0)$ & 1 \\
\hline 1 & $(1)$ & $\diamond(a \rightarrow b)$ & 0 & & $\square a \rightarrow \diamond b$ & (2) & 1 \\
\hline 1 & $(3)$ & $\square a$ & 2 & & $\Delta b$ & $(4)$ & 1 \\
\hline 1 & $(5)$ & $?_{\diamond}$ & 4 & & $b$ & $(12)$ & 1.1 \\
\hline 1.1 & $(7)$ & $a \rightarrow b$ & & 1 & $? \diamond$ & $(6)$ & 1.1 \\
\hline 1.1 & $(9)$ & $a$ & & 3 & $? \square / 1.1$ & $(8)$ & 1 \\
\hline 1.1 & $(11)$ & $b$ & & 7 & $a$ & $(10)$ & 1.1 \\
\hline
\end{tabular}

\begin{tabular}{|c|c|c|c|c|c|c|c|}
\hline & \multicolumn{3}{|c|}{$\mathrm{O}$} & \multicolumn{3}{|c|}{$\mathbf{P}$} & \\
\hline & & & & & $(\diamond a \rightarrow \square b) \rightarrow \square(a \rightarrow b)$ & $(0)$ & 1 \\
\hline 1 & $(1)$ & $\diamond a \rightarrow \square b$ & 0 & & $\square(a \rightarrow b)$ & $(2)$ & 1 \\
\hline 1 & $(3)$ & $? \square / 1.1$ & 2 & & $a \rightarrow b$ & $(4)$ & 1.1 \\
\hline 1.1 & $(5)$ & $a$ & 4 & & $b$ & $(10)$ & 1.1 \\
\hline 1 & $(7)$ & $\square b$ & & 1 & $\overline{\nabla a}$ & (6) & 1 \\
\hline 1.1 & $(9)$ & $b$ & & 7 & $? \square / 1.1$ & $(8)$ & 1 \\
\hline 1 & $(11)$ & $? \diamond$ & 6 & & $a$ & $(12)$ & 1.1 \\
\hline
\end{tabular}

DialKi $\neq$ DialK $\quad$ This can be shown with the following game, played according to both sets of rules. With the intuitionistic version the proponent cannot answer to (9) and loses, whereas with the standard ones, she can go further and revise her defence against (3). Hence DialK $\vDash \square(a \vee b) \rightarrow(\square a \vee \diamond b)$ but DialKi $\not \models \square(a \vee b) \rightarrow(\square a \vee \diamond b)$.

\begin{tabular}{|c|c|c|c|c|c|c|c|}
\hline & \multicolumn{3}{|c|}{$\mathrm{O}$} & \multicolumn{3}{|c|}{$\mathbf{P}$} & \\
\hline & & & & & $\square(a \vee b) \rightarrow(\square a \vee \diamond b)$ & $(0)$ & 1 \\
\hline 1 & (1) & $\square(a \vee b)$ & 0 & & $\square a \vee \diamond b$ & $(2)$ & 1 \\
\hline 1 & $(3)$ & $?$ & 2 & & $\square a$ & (4) & 1 \\
\hline 1 & $(5)$ & $? \square / 1.1$ & 4 & & & & \\
\hline 1.1 & $(7)$ & $a \vee b$ & & 1 & $? \square / 1.1$ & $(6)$ & 1 \\
\hline 1.1 & $(9)$ & $b$ & & 7 & $?$ & $(8)$ & 1.1 \\
\hline 1 & $\left(3^{\prime}\right)$ & $?$ & 2 & & $\Delta b$ & $(\mathbf{1 0})$ & 1 \\
\hline 1 & $(\mathbf{1 1})$ & $? \diamond$ & 10 & & $b$ & $(12)$ & 1.1 \\
\hline
\end{tabular}

(Here (3') is not a move but a repetition of (3) to let the reader see the attack the proponent answers to at round (10).) 


\section{References}

[1] van Benthem, Johan: 1991, 'Reflections on Epistemic Logic', Logique Es Analyse 133-134 (1991), 5-14.

[2] van Benthem, Johan: 2001, 'Logic in Games', Lecture Notes, ILLC Amsterdam.

[3] Celani, Sergio: 2001: 'Remarks on Intuitionistic Modal Logics', Divulgaciones Mathematicas 9(2), 137-147.

[4] Cozic, Mikaël: 2004, 'Epistemic models, Logical Monotony and Substructural Logics', Preprint. To appear in J. van Benthem et al, eds., The Age of Alternative Logics, Kluwer Academic Publishers, Dordrecht.

[5] Duc, Ho Ngoc: 1997, 'Reasoning about Rational, but not Logically Omniscient Agents', Journal of Logic and Computation 7(5), 633-648.

[6] Fitting, Melvin C.: 1969, Intuitionistic Logic Model Theory and Forcing, North Holland Publishing Company, Amsterdam-London.

[7] Hintikka, Jaakko: 1962, Knowledge and Belief, Reidel, Dordrecht.

[8] Kripke, Saul: 1965, 'Semantical Analysis of Intuitionistic Logic I', in Formal Systems ans Recursive Functions, North-Holland, Amsterdam, 92-130.

[9] Lorenzen, Paul \& Kuno Lorenz: 1978, Dialogische Logik, Darmstadt, WBG.

[10] Rahman, Shahid: 2003, 'Non-Normal Dialogics for a Wonderful World and More', Preprint. To appear in J. van Benthem et al, eds., The Age of Alternative Logics, Kluwer Academic Publishers, Dordrecht.

[11] Rahman, Shahid \& Laurent Keiff: 2004, 'On how to be a Dialogician', Preprint. To appear in D. Vandervecken, ed., Logic, Thought and Action, Kluwer, Dordrecht.

[12] Rahman, Shahid \& Helge Rückert: 1999, 'Dialogische Modallogik für T, B, S4 und S5', Logique et Analyse, 167-168, 243-282.

[13] Thomason, Richmond H.: 2000, 'Modelling the Beliefs of Other Agents', in J. Minker (ed.): 2000, Logic-Based Artificial Intelligence, Kluwer Academic Publishers, Dordrecht.

[14] Wolter, Frank \& Michael Zakharyaschev: 1999, 'Intuitionistic Modal Logic', in A. Cantini, E. Cesari \& P. Minari (eds.): 1999, Logic and Foundations of Mathematics, Kluwer, Dordrecht, 227-238. 